\title{
ON MEASURE SPACES WHERE EGOROFF'S THEOREM HOLDS
}

\author{
LÁSZLÓ ZSILINSZKY
}

\begin{abstract}
A measure space $(X, S, \mu)$ is called almost finite if $X$ is a union of a set of finite measure and finite many atoms of infinite measure. It is shown that Egoroff's Theorem for sequences of measurable functions holds if and only if the underlying measure space is almost finite. As a consequence we obtain several theorems on the interaction between convergences almost everywhere, almost uniform and in measure, respectively, with no preliminary conditions on the measure space $(X, S, \mu)$, thus extending results from [2],[4],[6],[10]. It is proved further that if $(X, S, \mu)$ is almost finite (is not almost finite) then $\phi: \mathbb{R} \rightarrow \mathbb{R}$ preserves almost uniform convergence and convergence in measure, respectively, if and only if $\phi$ is continuous (is uniformly continuous), thus augmenting a result of [3].
\end{abstract}

\section{INTRODUCTION}

Let $(X, S, \mu)$ be a measure space with $\mu(X)>0$. Denote by $\mathcal{M}$ the class of all $S$-measurable functions $f: X \rightarrow[-\infty,+\infty]$ that are finite almost everywhere (abbr. a.e.) on $X$.

A set $A \in S$ is called an atom if $\mu(A)>0$ and for any $B \subset A, B \in S$ either $\mu(B)=0$ or $\mu(A \backslash B)=0$. The measure space $(X, S, \mu)$ is said to be purely atomic ([5]) if it is decomposable into a countable union of atoms.

Let $f, f_{n} \in \mathcal{M}(n \in \mathbb{N})$. The concepts of convergence of the sequence $\left\{f_{n}\right\}_{n=1}^{\infty}$ to $f$ almost everywhere (denoted $f_{n} \stackrel{a . e}{\longrightarrow} f$ ), almost uniformly $\left(f_{n} \stackrel{a . u .}{\longrightarrow} f\right)$ and in measure $\left(f_{n} \stackrel{\mu}{\longrightarrow} f\right)$, respectively are well-known, their mutual connections are well established and belong to stock theorems of Measure Theory (cf.[5]).

Some of these theorems hold in case $\mu(X)<\infty$, but need not hold in general. Such theorems are e.g. the well-known Egoroff Theorem ([1]) asserting that convergence a.e. implies almost uniform convergence, or a theorem of Lebesgue asserting that convergence a.e. implies convergence in measure, which is actually a consequence of Egoroff's Theorem since almost uniform convergence implies convergence in measure without restrictions on $\mu$ ([5],p.92). For examples showing that these theorems need not hold in infinite measure spaces, see [5] (pp.90,94).

Various papers deal with diverse generalization of Egoroff's Theorem e.g. for collections of functions instead of sequences (cf.[7],[9],[11]), however it is also possible to extend the classical theorem (see [2]). It is the purpose of this paper to characterize measure spaces where Egoroff's Theorem holds and draw consequences

1991 Mathematics Subject Classification. 28A20; Secondary 40A30.

Typeset by $\mathcal{A}_{\mathcal{M S}}$ - $\mathrm{T}_{\mathrm{EX}}$ 
on the interaction between the mentioned types of convergence without preliminary conditions on $(X, S, \mu)$, thus extending results from [10],[6],[4]. As a consequence we will characterize functions which preserve the investigated modes of convergence, thus augmenting a theorem of [3].

It turns out that the appropriate measure space is one that can be decomposed into the union of a set of finite measure and finitely many atoms of infinite measure. We will refer to it as an almost finite measure space. There is a characterization of these spaces via collections of pairwise disjoint measurable sets:

Theorem 1. The following are equivalent:

(i) $(X, S, \mu)$ is almost finite,

(ii) $\lim _{n \rightarrow \infty} \mu\left(X_{n}\right)=0$ for each sequence $\left\{X_{n}\right\}_{n=1}^{\infty}$ of pairwise disjoint $S$ measurable sets.

Proof. (i) $\Rightarrow$ (ii) Let $\left\{X_{n}\right\}_{n=1}^{\infty}$ be a sequence of pairwise disjoint $S$-measurable sets. Without loss of generality we may assume that $\mu\left(X_{n}\right)>0$ for all $n \in \mathbb{N}$. Then, in view of (i),

$$
\sum_{n \geq n_{0}} \mu\left(X_{n}\right)=\mu\left(\bigcup_{n \geq n_{0}} X_{n}\right)<+\infty
$$

for some $n_{0} \in \mathbb{N}$, thus $\lim _{n \rightarrow \infty} \mu\left(X_{n}\right)=0$.

(ii) $\Rightarrow$ (i) According to Zorn's Lemma there is a maximal element $\mathcal{F}$ in the class of all families of pairwise disjoint $S$-measurable sets of positive finite measure. In view of (ii) the set $\mathcal{F}_{j}=\left\{E \in \mathcal{F} ; \mu(E)>\frac{1}{j}\right\}$ is finite for all $j \in \mathbb{N}$, thus $\mathcal{F}=\bigcup_{j=1}^{\infty} \mathcal{F}_{j}$ is countable, say $\mathcal{F}=\left\{F_{1}, \ldots, F_{k}, \ldots\right\}$ where $F_{k} \in S(k \in \mathbb{N})$. Consequently, $F=\bigcup_{k=1}^{\infty} F_{k} \in S$

To show that $\mu(F)<+\infty$ it suffices to observe that otherwise we can find an increasing sequence $\left\{k_{i}\right\}_{i=1}^{\infty}$ of natural numbers such that

$$
\mu\left(\bigcup_{l=k_{i}}^{k_{i+1}} F_{l}\right) \geq 1 \text { for all } i \in \mathbb{N} .
$$

Since $\mathcal{F}$ is maximal, the measurable subsets of $G=X \backslash F$ are either nullsets or sets of infinite measure. If these subsets of $G$ contained infinitely many different sets of infinite measure, then $G$ would contain a sequence of pairwise disjoint measurable sets of infinite measure, which contradicts (ii).

\section{Main Results}

First we prove an extension of the theorem of Egoroff and Lebesgue, respectively:

Theorem 2. The following are equivalent:

(i) $(X, S, \mu)$ is almost finite,

(ii) for any $f, f_{n} \in \mathcal{M}, f_{n} \stackrel{\text { a.e. }}{\longrightarrow} f$ implies $f_{n} \stackrel{\text { a.u. }}{\longrightarrow} f$,

(iii) for any $f, f_{n} \in \mathcal{M}, f_{n} \stackrel{\text { a.e. }}{\longrightarrow} f$ implies $f_{n} \stackrel{\mu}{\longrightarrow} f$.

Proof. (i) $\Rightarrow$ (ii) Suppose that $X=F \cup G$ where $\mu(F)<+\infty$ and $G$ is a finite union of atoms of infinite measure. Let $f, f_{n} \in \mathcal{M}(n \in \mathbb{N})$ such that $f_{n} \stackrel{\text { a.e. }}{\longrightarrow} f$. 
Measurable functions are constant a.e. on atoms, consequently $\left\{f_{n}\right\}_{n=1}^{\infty}$ converges uniformly a.e. (hence also almost uniformly) on $G$ to $f$. On the other hand on $F$ Egoroff's Theorem applies.

(ii) $\Rightarrow$ (iii) It suffices to notice that almost uniform convergence implies convergence in measure ([5],p.92).

(iii) $\Rightarrow$ (i) Suppose that $(X, S, \mu)$ is not almost finite. Then by Theorem 1 there exists a sequence $\left\{X_{n}\right\}_{n=1}^{\infty}$ of pairwise disjoint $S$-measurable sets such that $\lim _{n \rightarrow \infty} \mu\left(X_{n}\right) \neq 0$. Put $f \equiv 0$ and $f_{n}=\chi_{X_{n}}$ for all $n \in \mathbb{N}\left(\chi_{A}\right.$ stands for the characteristic function of $A \subset X)$. Then $f_{n} \stackrel{\text { a.e. }}{\longrightarrow} f$ but $f_{n} \stackrel{\mu}{\leftrightarrow} f$.

Corollary 1. The following are equivalent:

(i) $(X, S, \mu)$ is almost finite,

(ii) for any $f, f_{n} \in \mathcal{M}, f_{n} \stackrel{\text { a.e. }}{\longrightarrow} f$ iff $f_{n} \stackrel{a . u .}{\longrightarrow} f$.

Proof. It suffices to observe that almost uniform convergence always implies convergence almost everywhere and apply Theorem 2 .

Corollary 2. The following are equivalent:

(i) $(X, S, \mu)$ is almost finite and purely atomic,

(ii) for any $f, f_{n} \in \mathcal{M}, f_{n} \stackrel{\text { a.e. }}{\longrightarrow} f$ iff $f_{n} \stackrel{\mu}{\longrightarrow} f$.

Proof. It easily follows from our Theorem 2 and from Theorem 1 in [10] (cf. also $[6],[4])$.

Further we prove a generalization of a theorem of Riesz ([8]) about the interplay between convergence a.e. and in measure, respectively:

Theorem 3. The following are equivalent:

(i) $(X, S, \mu)$ is almost finite,

(ii) for any $f, f_{n} \in \mathcal{M}, f_{n} \stackrel{\mu}{\longrightarrow} f$ iff every subsequence $\left\{f_{n_{k}}\right\}_{k=1}^{\infty}$ of $\left\{f_{n}\right\}_{n=1}^{\infty}$ contains a subsequence $\left\{f_{n_{k_{j}}}\right\}_{j=1}^{\infty}$ such that $f_{n_{k_{j}}} \stackrel{\text { a.e. }}{\longrightarrow} f$.

Proof. (i) $\Rightarrow$ (ii) Suppose that $(X, S, \mu)$ is almost finite. Suppose that there exists a sequence $\left\{f_{n}\right\}_{n=1}^{\infty}$ every subsequence of which contains a subsequence converging a.e. on $X$ to $f \in \mathcal{M}$, while $f_{n} \stackrel{\mu}{\leftrightarrow} f$. Then $\left\{f_{n}\right\}_{n=1}^{\infty}$ has a subsequence $\left\{f_{n_{k}}\right\}_{k=1}^{\infty}$ such that for some $\varepsilon_{0}, \delta_{0}>0$

$$
a_{k}=\mu\left(\left\{x \in X ;\left|f_{n_{k}}(x)-f(x)\right| \geq \varepsilon_{0}\right\}\right) \geq \delta_{0} \text { for all } k \in \mathbb{N} .
$$

According to the assumptions there is a subsequence $\left\{f_{n_{k_{j}}}\right\}_{j=1}^{\infty}$ of $\left\{f_{n_{k}}\right\}_{k=1}^{\infty}$ converging a.e. to $f$, thus by Theorem 2 it converges also in measure to $\mathrm{f}$ on $X$. Consequently $a_{k_{j}} \rightarrow 0(j \rightarrow \infty)$, which contradicts (1).

(ii) $\Rightarrow$ (i) Assume that $(X, S, \mu)$ is not almost finite. Then in view of Theorem 2 there exists a sequence converging a.e. on $X$ but not in measure, hence (ii) fails to hold.

Now we turn to investigating the question of preservation of measurables functions under composition (cf.[3]). More precisely, if $M$ denotes a mode of convergence for a sequence of measurable functions then we find necessary and sufficient 
conditions for the function $\phi: \mathbb{R} \rightarrow \mathbb{R}$ to satisfy the following implication:

$$
\text { for any } f, f_{n} \in \mathcal{M}, f_{n} \stackrel{M}{\longrightarrow} f \text { implies } \phi \circ f_{n} \stackrel{M}{\longrightarrow} \phi \circ f \text {. }
$$

The question is easy for convergence a.e. (see [3], Theorem 1):

Theorem 4. The function $\phi$ preserves convergence almost everywhere iff $\phi$ is continuous.

For the remaining types of convergence Theorem 2 in [3] gives only a partial solution. A fuller answer is as follows:

\section{Theorem 5.}

(i) Suppose that $(X, S, \mu)$ is almost finite. Then $\phi$ preserves almost uniform convergence and convergence in measure, respectively iff $\phi$ is continuous.

(ii) Suppose that $(X, S, \mu)$ is not almost finite. Then $\phi$ preserves almost uniform convergence and convergence in measure, respectively iff $\phi$ is uniformly continuous.

Proof. (i) For almost uniform convergence the theorem follows from Corollary 1 and Theorem 4 . Further Theorems 3 and 4 yield the desired result for convergence in measure.

(ii) In view of Theorem 1 there exists a sequence $\left\{X_{n}\right\}_{n=1}^{\infty}$ of pairwise disjoint $S$-measurable sets such that

$$
\lim _{n \rightarrow \infty} \mu\left(X_{n}\right) \neq 0
$$

The sufficiency of the condition is clear (see [3],Theorem 2). Conversely, suppose that $\phi$ is not uniformly continuous. Then there are scalars $z_{n}, z_{n}^{\prime}$ such that $\left|z_{n}-z_{n}^{\prime}\right|<\frac{1}{n}$ and $\left|\phi\left(z_{n}\right)-\phi\left(z_{n}^{\prime}\right)\right| \geq \varepsilon_{0}$ for some $\varepsilon_{0}>0$. Define the functions

$$
f_{n}=z_{n}^{\prime} \chi_{X_{n}}+\sum_{k \neq n} z_{k} \chi_{X_{k}}(n \in \mathbb{N}) \text { and } f=\sum_{k=1}^{\infty} z_{k} \chi_{X_{k}} .
$$

Then evidently $\left\{f_{n}\right\}_{n=1}^{\infty}$ tends uniformly to $f$, consequently it converges to $f$ almost uniformly and in measure as well.

On the other hand $\left\{x \in X ;\left|\phi \circ f_{n}(x)-\phi \circ f(x)\right| \geq \varepsilon_{0}\right\}=X_{n}$ for all $n \in \mathbb{N}$, thus in view of $(2) \phi \circ f_{n} \stackrel{\mu}{\leftrightarrow} \phi \circ f$, hence $\phi \circ f_{n} \stackrel{a . u .}{\rightarrow} \phi \circ f$.

\section{REFERENCES}

[1] D.F.Egoroff, Sur les suites des fonctions mesurables, A. R. Acad. Paris 152 (1911), 244-246.

[2] R.G.Bartle, An extension of Egorov's theorem, Amer. Math. Monthly 87 (1980), 628-633.

[3] R.G.Bartle and J.T.Joichi, The preservation of convergence of measurable functions under composition, Proc. Amer. Math. Soc. 12 (1961), 122-126.

[4] J.I.Gribanov, Remarks on convergence almost everywhere and in measure, Comment. Math. Univ. Carolin. 73 (1966), 297-300 (Russian).

[5] P.R.Halmos, Measure Theory, D. van Nostrand, New York, 1950.

[6] E.Marczewski, Remarks on the convergence of measurable sets and measurable functions, Coll. Math. 3 (1955), 118-124. 
[7] T.Neubrunn, Almost uniform convergence for continuous parameters, Math. Slovaca 28 (1978), 321-328.

[8] F.Riesz, Sur les suites de fonctions mesurables, C. R. Acad. Sci. Paris 148 (1909), 1303-1305.

[9] E.P.Rozycki, On Egoroff's theorem, Fund. Math. 56 (1965), 289-293.

[10] R.J.Tomkins, On the equivalence of modes of convergence, Canad. Math. Bull. 16 (1973), 571-575.

[11] E.Zakon, On almost uniform convergence of families of functions, Canad. Math. Bull. 7 (1964), 45-48.

Mathematics Department, College of Education, Farská 19, 94974 Nitra, SloVAKIA

E-mail address: zsili@unitra.sk 